\title{
Vorwort der russischsprachigen Ausgabe
}

Im Jahre 1968 waren $40 \mathrm{~J}$ ahre seit der Entdeckung des RAMAN-Effektes vergangen. Die Raman-Spektroskopie hat sich in dieser Zeit einen festen Platz unter den vielfältigen Analysen- und Strukturbestimmungsmethoden erobert. Ihre Bedeutung ist ständig im Wachsen begriffen. Eine sprunghafte Entwicklung der RAMaN-Spektroskopie wurde in den letzten Jahren durch die Vervollkommnung der experimentellen Technik, inslesondere durch den Einsatz von Lasern als Erregerlichtquellen, hervorgerufen. Durch die Einführung neuer Untersuchungsmethoden ist es möglich geworden, die bis vor wenigen Jahren übliche Beschränkung der Anwendung des RAMAN-Effektes auf durchsichtige Substanzen zu überwinden, und auch RAMAN-Spektren von Kristallpulvern und absorbierenden Substanzen zu erhalten. In ähnlicher Weise sind die Anforderungen an die Menge der Streusubstanz bedeutend gesunken. Alles das hat dazu beigetragen, daß sich die Klasse der für RAMANspektroskopische Untersuchungen geeigneten Substanzen beachtlich erweitert hat.

Parallel zur Ausdehnung des praktischen Anwendungsgebietes des RAMANEffektes zeichnet sich in den letzten Jahren eine verstärkte Untersuchung seiner physikalischen Natur ab. Besonderes Interesse rief die Entdeckung einer neuen Erscheinung der nichtlinearen Optik, der stimulierten RAMAN-Streuung, hervor. Diese Entdeckung warf eine Reihe von Fragen auf, die die Natur des RamanEffektes und dessen Stellung unter verwandten physikalischen Vorgängen betreffen. Im Zusammenhang mit der sich erweiternden Anwendung des RAMaNEffektes und neuen Entdeckungen auf diesem Gebiet wurde die Aufmerksamkeit eines großen Kreises von Physikern und Chemikern auf den Raman-Effekt gelenkt. Obwohl der RAMAN-Spektroskopie eine umfangreiche Literatur gewidmet ist, gibt jedoch nur Placzer in seiner Monographie eine hinreichend tiefe und systematische Behandlung der allgemeinen Probleme dieser Erscheinung. Dieses seinem Umfang nach kleine und relativ schwer zu lesende Buch erschien in russischer Sprache im Jahre 1935 und ist längst zu einer bibliophilen Seltenheit geworden. Andere auf diesem Gebiete veröffentlichte Bücher sind vorwiegend derMeßmethodik gewidmet oder umfassen nur einen relativ kleinen, die Anwendung der Raman-Srektroskopie in der Chemie betreffenden Problemkreis. Daneben existiert eire Reihe von Arbeiten zur Berechnung von Molekülschwingungsspektren in denen jedoch die Behandlung des RAMAN-Effektes selbst nur eine zweitrangige Stellung einnimmt.

Mit dem vorliegenden Buch wird der Versuch unternommen, die in der Literatur vorhandene Lücke zu schließen, und eine systematische Darlegung der für das Verständnis des physikalischen Wesens des RamaN-Effektes und seines 


\section{Vorwort der russischsprachigen Ausgabe}

Zusammenhangs mit anderen optischen Vorgängen notwendigen Grundfragen zu geben. Darüber hinaus soll das Buch einen Eindruck über die vielfältigen Anwendungsmöglichkeiten des Raman-Effektes bei der Untersuchung von Substanzeigenschaften vermitteln. Hierzu gehören vor allem Aussagen über den chemischen Aufbau, die geometrische Konfiguration und die zahlreichen geometrischen, dynamischen und elektrooptischen Parameter der Moleküle. Spezielle Kapitel sind den RAMAN-Spektren von Kristallen und dem stimulierten RaMaN-Effekt gewidmet.

Das Buch wendet sich an einen breiten Kreis von Wissenschaftlern, die sich mit dem RAMAN-Effekt beschäftigen, jedoch nicht über Spezialkenntnisse auf diesem Gebiet verfügen. Die Darstellung einer Reihe von Problemen wird erforderlichenfalls durch erläuterndes Material ergänzt. In einigen Fällen werden im geringen Umfange notwendige mathematische Angaben gemacht. Es ist klar, daß dabei keine Strenge und Systematik der Darstellung angestrebt werden konnte. Auf die Berechnung von Molekülschwingungen wird nur soweit eingegangen, wie das für das Verständnis der hier behandelten Probleme erforderlich ist.

Fragen der experimentellen Technik werden nur in einigen Fällen im Zusammenhang mit wenig bekannten oder neuen Untersuchungsmethoden beschrieben. Die im Buch angeführten experimentellen Daten dienen hauptsächlich zur Illustration der Grundprobleme und stellen deshalb nur einen kleinen Ausschnitt aus dem vorliegenden experimentellen Material dar. Die Zitierung der einschlägigen Literatur erhebt ebenfalls keinen Anspruch auf Vollständigkeit, selbst dann nicht, wenn eine Frage relativ ausführlich dargestellt wird.

Abschließend möchte ich W.S. GoRELIK für seine wertvolle Hilfe bei der Abfassung der Paragraphen 18 bis 21 danken sowie T. I. Kusnezowa, W. A. Subow, L. A. Schelepin und I. K. Schuwalow für Diskussionen über einige Abschnitte dieses Buches. W.P. Sotschelnikowa danke ich für ihre große Hilfe bei der Anfertigung des Manuskriptes.

Der Autor ist JA.S. Boвowitsch und CH. E. Sterin für die kritische Durchsicht des Manuskriptes und für wertvolle Hinweise zu Dank verpflichtet.

M. M. Suschtschinskij 